\title{
Low Cost, High Power Factor, Dimmable, Monolithic AC-Direct LED Driver with on-Chip Step-Dimmer for Outdoor Lighting Applications
}

\author{
Van Ha Nguyen, Sanguk Nam, Kilsoo Seo, Yongsu Park, and Hanjung Song
}

\begin{abstract}
In this paper, we develop a monolithic AC LED driver IC which can drive and dim high voltage LED strings directly from the $220 \mathrm{~V} / 60 \mathrm{~Hz}$ commercial AC power. The proposed AC LED driver delivers a best-in-class dimming performance via a simple concept with a step-dimmer, while maintaining a very PF by using a self-adapive power processing circuit. In order to verify the performance of the proposed scheme, a 4.4W, 8-string AC-directly-power LED driver was designed and simulated using $0.35 \mathrm{um}$ BCD Magnachip process technology. The simulated results show a PF $>0.99$ with a THD $<7 \%$ under different dimming modes. The high level of integration and low component count result in a cost-effective solution which is especially suitable for use in outdoor lighting applications.
\end{abstract}

Index Terms-AC-direct LED driver, adaptive power processing circuit, dimmable LED driver, self-adaptive.

\section{INTRODUCTION}

In outdoor lighting applications where the effects of flicker at $120 / 100 \mathrm{~Hz}$ from commercial AC mains can be neglected, AC-powered LED drivers are more attractive than switch-mode LED drivers since they simplify the design as well as reduce the cost of lighting module [1-[3]. Recently, there have been studies of high PF, low THD AC-directly-powered LED drivers reported in the literature [4]-[6]. Although these AC LED drivers can achieve a very high performance, they also have several shortcomings. With the design proposed in [4], it is a simplest and most cost-effective scheme since the design is constructed with a lowest number of components; however, as the number of LED strings increases, the design shows a difficulty of realizing the switching function. Another design reported in [5] shows a high PF with a dimming feature; however it is very complicated in implementing the design with expensive blocks which degrades its robustness. As the most effective design, the technique reported in [6] can gain a high very

Manuscript received April 20, 2015; revised August 20, 2015. This work was supported by the Human Resource Training Program for Regional Innovation and Creativity through the Ministry of Education and National Research Foundation of Korea (NRF-2014H1C1A1066686) and Basic Science Research Program through the National Research Foundation of Korea (NRF) funded by the Ministry of Education, Science and Technology (2012-0002777)

Van Ha Nguyen, Sanguk Nam, and Hanjung Song are with the Department of Nannoscience and Engineering, Inje University, South Korea (e-mail: hjsong@inje.ac.kr).

Kilsoo Seo is with the Korea Electrolytic Research Institute (KERI), South Korea.

Youngsu Park is with the Falcuty of Electrical and Electronic Engineering, Chung Cheong University, South Korea. high PF and a low THD using self-adaptive soft-switching method without any additional control circuit as well as can be customized to balance the requirements of performance and cost. However, with these AC LED drivers, dimming function using triac dimmer can be challenging. All of these designs cannot perform properly with the conventional triac dimmer as in switch-mode LED drivers because of the two main problems: 1) Degrade the PF due to current chopping, 2) Increase the flicker because of the dead zone of LED current which might result in negative effects on the health [3]. Therefore, a new and effective design of a dimmable AC-direct LED driver for outdoor lighting applications is needed. In this paper, an on-chip step-dimmer using analog dimming method in accompany with the novel self-adaptive power processing core reported in [6] is proposed for regulating simultaneously LED brightness as well as maintaining a high power factor and a low total harmonic distortion via a simple concept. Furthermore, there is no need of any external passive components, thereby enhancing the lifespan of the overall LED module as well as achieving a solution of a fully integrated circuit of the design.

\section{Proposed Multi-STRING AC-Direct LED Driver WITH ON-CHIP STEP-DIMMER}

\section{A. System Design and Fundamental Operation}

As depicted in Fig. 1, the proposed dimmable AC-direct LED driver consists of a bridge, a self-adaptive power processing circuit based on the design reported in [6] that drives multiple LED strings directly from the AC mains, a start-up circuit, a low voltage regulator LDO, and a step-dimming circuit. The output of the step-dimmers is connected in series with the power processing circuit to regulate the LED current and brightness.

Before entering in details of design, it is noticed that a high voltage $30 \mathrm{~V} / 20 \mathrm{~mA}$ LED type was utilized in the proposed design. In order to gain high PF and low THD, the long series-connected LED string is divided into substrings [5]. With $220 \mathrm{~V}$ (RMS) of input voltage and $30 \mathrm{~V} / 20 \mathrm{~mA}$ of the utilized LED type, 10 LED strings can be adopted in order to gain the highest PF. However, considering a small margin when the last LED string conducts and the increase of forward voltage of LED strings when the LED current increases, 8 LED strings is chosen. In the power processing circuit, the low voltage components are isolated from the high voltage of the AC mains by high voltage transistors HM1-HM8. There are eight modes for 8 LED strings within a half of operating cycle. Referring to the power processing 
circuit shown in Fig. 1 and the theoretical analysis of the self-adaptive power processing circuit reported in [6], the operating principle of this circuit can be fairly easy to understand and is summarized as following: When the input voltage $v_{\text {in }}$ increase to values higher than the sum of the first $k$ LED strings, the $k^{\text {th }}$ current regulation element $\operatorname{LCR}(\mathrm{k})$ which consists of one op-amp, transistor $M_{i}$ and one resistor $R_{i}$ is turned on and it conducts the LED current of the first $k$ LED strings. The LCRs are turned on in a sequence and this process repeats in the next cycles. Additionally, it is noticeable that the power processing core owns a self-adaptive mechanism; hence, there is no need of any additional controller as in the designs reported in $[4,5]$. Furthermore, since only one LCR turns on at a certain period, the loss power is minimized in order to increase the power efficiency.

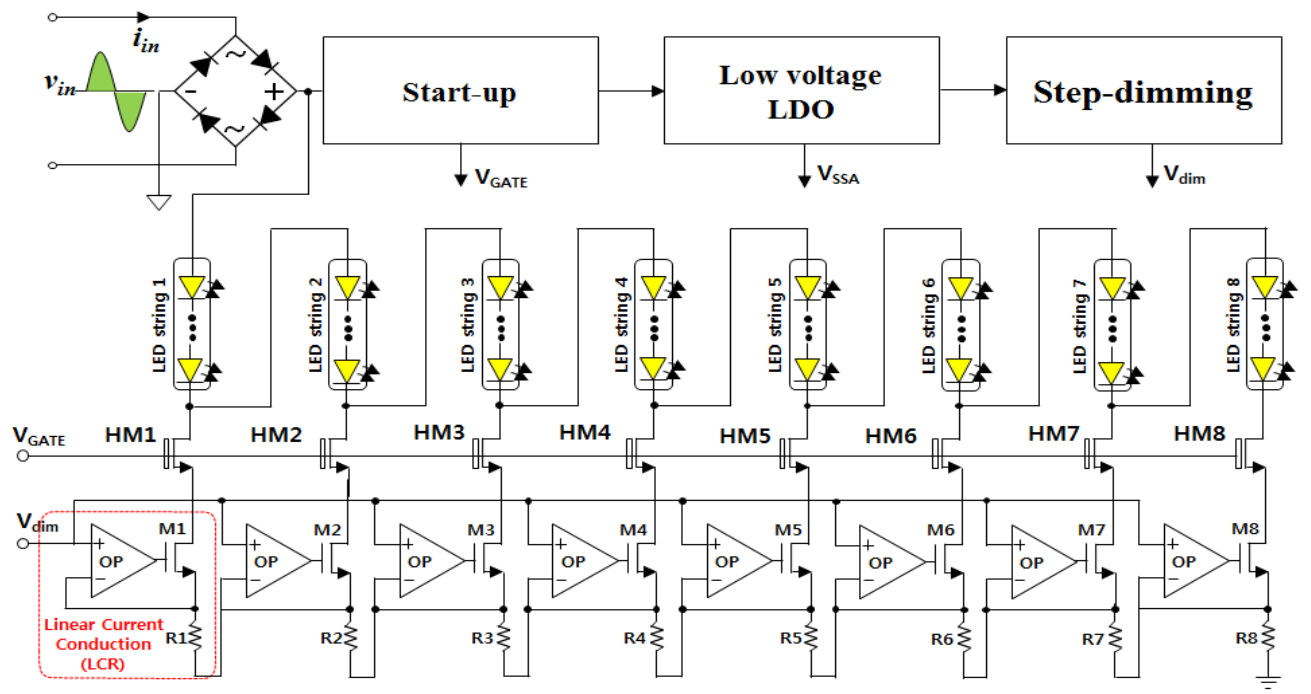

Fig. 1. Schematic diagram of the proposed dimmable AC-powered LED driver.
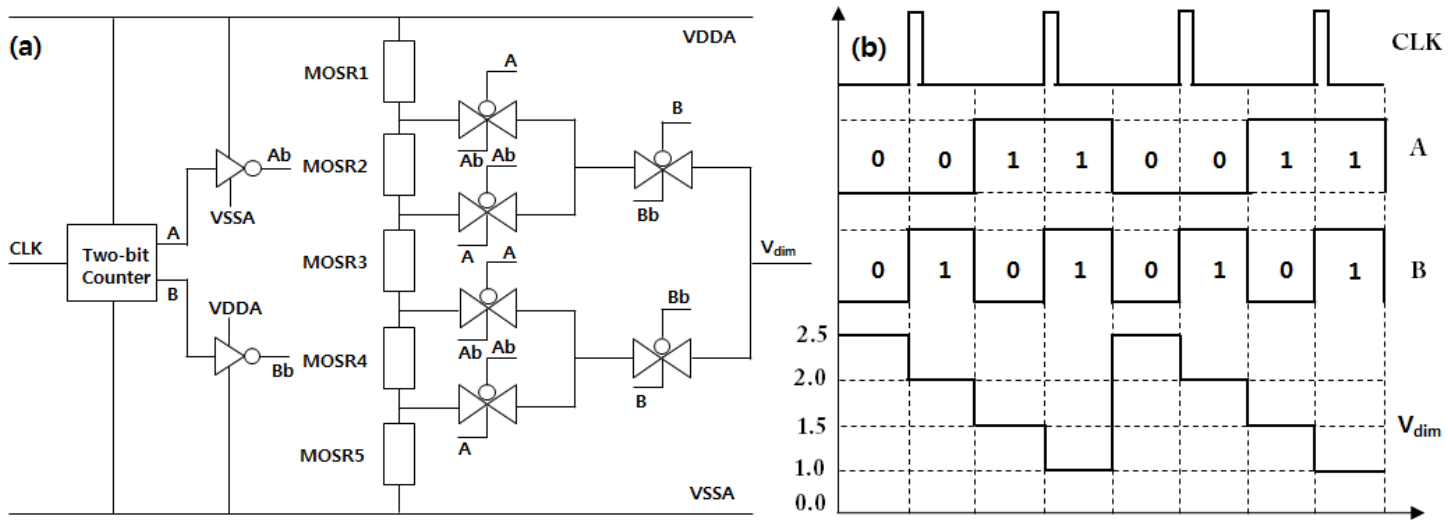

Fig. 2. The proposed step-dimmer. (a) Schematic. (b) Operation.

\section{B. A New Step-Dimmer with Amplitude Modulation Method}

To adjust the brightness of the LED lamps, a step-dimmer is connected in series with the power processing core to regulate the LED current and brightness as shown in Fig. 1. Since the bias current for the sub-circuits including start-up circuit, LDO and dimming circuit are small as compared to the LED current and can be neglected; the input current $i_{\text {in }}$ approximates to the LED current $i_{L E D}$ which is calculated as the sum of the current elements of LCRs $i_{i}$ and can be expressed as:

$$
i_{i n}=i_{L E D}=i_{1}+i_{2}+\ldots+i_{n}=\sum_{i=1}^{8} i_{i}
$$

with:

$$
i_{i}=\frac{V_{\mathrm{dim}}}{R_{i}}
$$

As shown in Fig. 1, it is easily realized that the LED current is simply regulated by monitoring the dimming voltage $V_{\text {dim }}$ of LCRs. Additionally. as explained about the self-adaptive mechanism of the power processing circuit in [6], a step-like sinusoidal of input current can be achieved; hence, therefore, all the LED strings are dimmed simultaneously to maintain the PF and THD. Because of this, the driver now is considered like a resistive load $R_{\text {eq_driver }}$ from the view of the AC mains. Assuming that the input current of AC LED driver is the ideal sinusoidal waveform, so it can be expressed as follows:

$$
i_{\text {in }}=I_{0} \sin \omega t=\frac{V_{\mathrm{dim}}}{R_{\text {eq_driver }}} \sin \omega t
$$

where $I_{o}$ is the peak value the input current. Now, it is clear that the LED current depends on the value of dimming voltage $V_{\text {dim }}$ generated from the step-dimmer. 

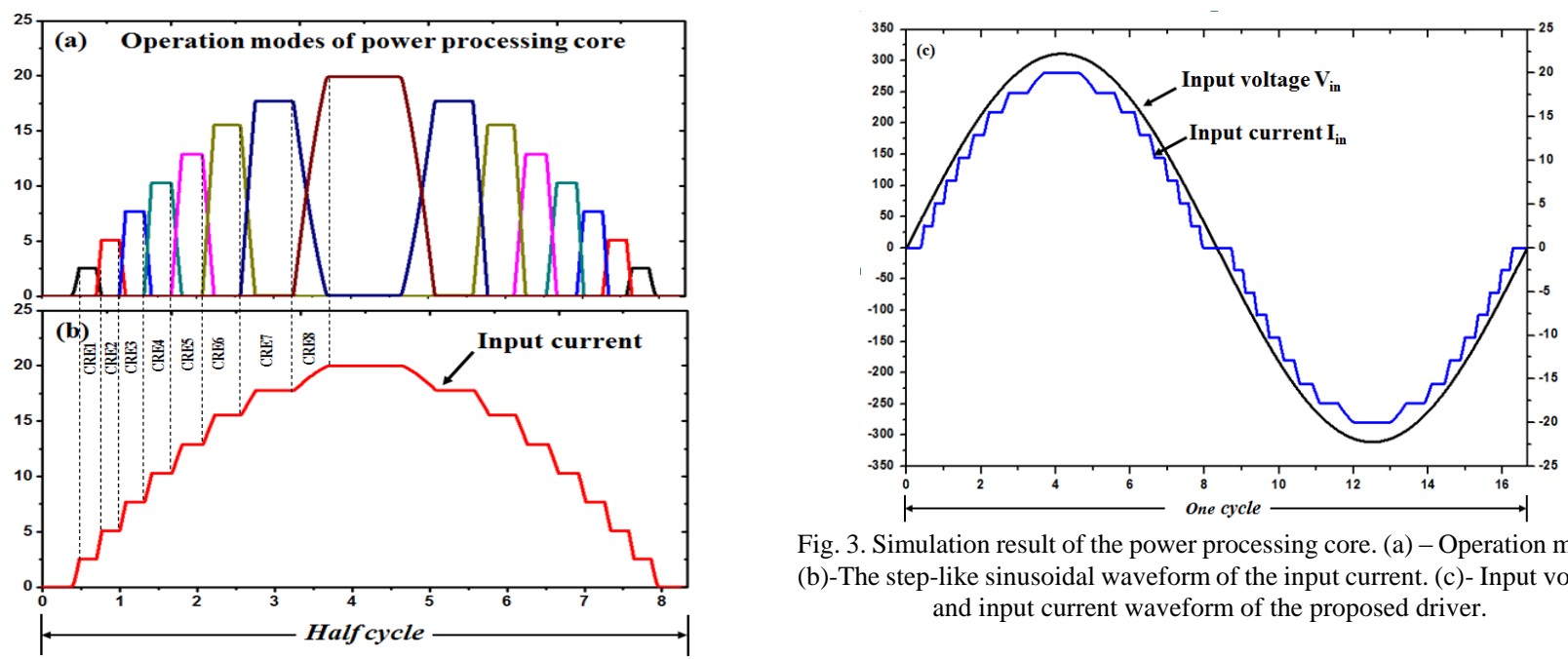

Fig. 3. Simulation result of the power processing core. (a) - Operation modes. (b)-The step-like sinusoidal waveform of the input current. (c)- Input voltage and input current waveform of the proposed driver.

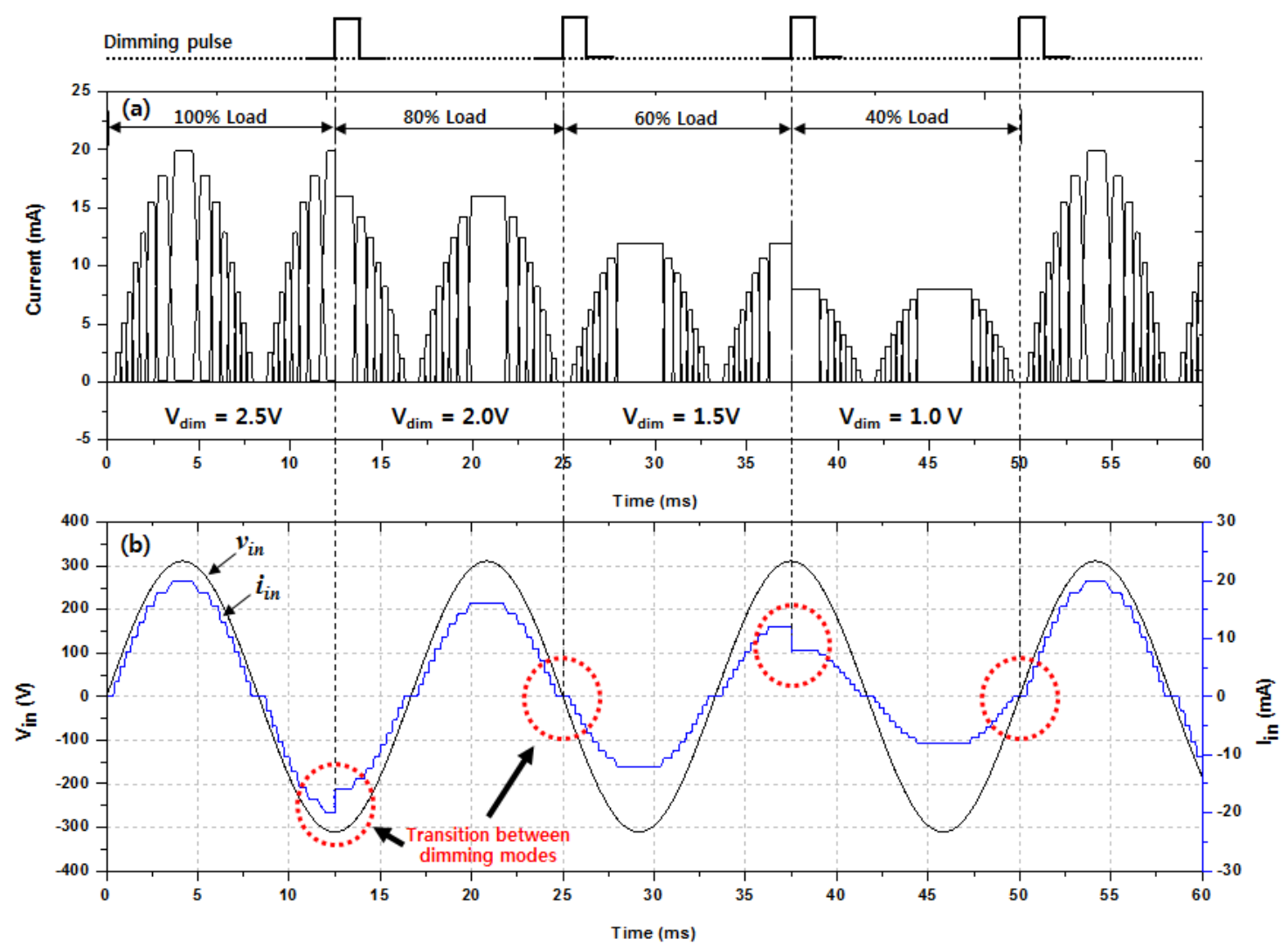

Fig. 4. Simulation result of AC LED Driver when a dimming step is applied.

The step-dimmer proposed in this work is constructed using simple digital cells including a 2-bit counter, 6 transmission gates, 2 inverters and a voltage divider to control the dimming voltage $V_{\text {dim }}$ as shown in Fig. 2(a). Under a pulse from the external switch, the counter outputs different states in sequence and decides the ON/OFF states of the transmission gates to generate the desired value of dimming signal as shown in Fig. 2 (b). An integrated voltage divider MOSR1-MOSR5using technique reported in [9] is utilized in lieu of a resistor-based voltage divider which reduces the chip size as well as reduces the impact of fabrication process. To expand the range of dimming, the counter with a higher resolution can be used. In combining with the self-adaptive power processing, it is easy to customize the design in a high $\mathrm{PF}$ required by IEC 61000-3-2 Class $\mathrm{C}$ via choosing the number of LED strings and the smooth of dimming based on choosing the resolution of the step-dimmer.

\section{Simulation ReSUlts}

To verify the feasibility of the proposed scheme, the circuit is designed and simulated using 0.35 um $700 \mathrm{~V}$ BCD technology that provides high voltage devices. In order to reduce the nonlinearity that may cause a reduction of the efficacy in Lumens per Watt if the operating current of LEDs exceeds the linear range, the full load of LED is set at $20 \mathrm{~mA}$ (peak value) instead of $20 \mathrm{~mA}$ (RMS value). In the simulation, the value of Vdim was chosen in range of 1 to $2.5 \mathrm{~V}$ with a step of $0.5 \mathrm{~V}$ as shown in Fig. 2(b). The start-up circuit was designed to bias the power transistor HM1-HM8 with a value of $7.5 \mathrm{~V}$ for $\mathrm{V}_{\mathrm{GATE}}$.

Fig. 3 shows the waveforms of the utilized power processing core with 8 LED strings. As seen in Fig. 3(a), the operation of the power processing core is depicted with a 
smooth transition between LCRs. Fig. 3(b) shows the input current waveform in a half of cycle that is the sum of current elements of LCRs while the waveform of the input current and the input voltage are shown in Fig. 3(c). It is clearly seen that the input current $i_{i n}$ is in a nearly-sinusoidal waveform and always is in phase with the input voltage $v_{i n}$, so a high PF and low THD is ensured. Fig. 4 shows the input current waveform at difference diming modes when the dimming signal is applied. The transition between dimming modes is smooth without any current glitches. As a result, the dimming function does not affect to the performance of the power processing core driver and the high PF and low THD are maintained at all dimming conditions.

Finally, the performance of the circuit against the dimming was calculated in PF and THD against the dimming modes at $25 \%, 50 \%, 75 \%$ and $100 \%$ dimming levels, respectively. The $\mathrm{PF}$ was found at 0.997 for $25 \%$ dimming level and 0.9984 for $100 \%$ dimming level while the THD was found at $6.7 \%$ for $25 \%$ dimming level and $5.6 \%$ for $100 \%$ dimming level which comply the IEC 61000-3-2 class C.

\section{CONCLUSION}

In this paper, an un-expensive, high power factor, dimmable, monolithic AC LED driver IC with a simple and effective dimming method has been presented. The simulation via $0.35 \mathrm{um}-700 \mathrm{~V}$ BCD high voltage Magnachip process of a $4.4 \mathrm{~W}, 8$-string AC-direct LED driver was realized to verify the feasible of the proposed scheme. The gain PF and THD was found around 0.998 and 6\%, respectively, at all dimming modes that satisfies international standard IEC 61000-3-2 Class C. The proposed circuit can be implemented at lower cost than the switch-mode LED drivers, thus well suited for use in LED lamps for outdoor lighting applications.

\section{REFERENCES}

[1] C. Cheng, C. Chang, T. Chung and F. Yang, "Design and implementation of a single-stage driver for supplying an LED street-lighting module with power factor corrections," IEEE Transactions on. Power Electronics. vol. 30, no. 2, pp. 956-966, 2015.

[2] M. Arias, D. G. Lamar, J. D. Sebastian, and A. A. Diallo, "High-efficiency LED driver without electrolytic capacitor for street lighting," IEEE Transactions on Industry Applications, vol. 49, no. 1, pp.127-137, 2013.

[3] J. Lam and P. K. Jain, "A novel isolated electrolytic capacitor-less single-switch AC-DC offline LED driver with power factor correction," IEEE Applied Power Electronics Conference and Exposition (APEC 14), pp. 1356 - 1361, 2014.

[4] K. S. Seo, V. H. Nguyen, J. W. Jung, J. S. Park, and H. J. Song, "Multi-string AC-powered LED driver with current regulation reduction based on simple circuitry," IEICE Electronics Express (ELEX), vol. 11, no. 19, p. 20140810, 2014.

[5] K. I. Hwu and W. C. Tu, "Dimmable driver for light-emitting diode with total harmonic distortion improved," IET Power Electronics, vol. 5, no. 1, pp. 59-67, 2012.

[6] N. Ning, W. B. Chen, D. J. Yu, C. Y. Feng and C. B. Wang, "Self-adaptive load technology for multiple-string LED drivers," Electronics Letters, vol. 49, no. 18, pp. 1170-1171, 2013.

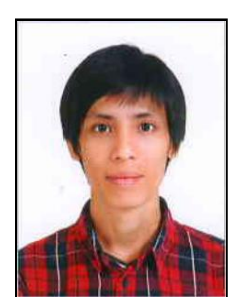

Van Ha Nguyen received the B.E. and M.S. degrees in electrical-electronics engineering and nano device from University of Technology and Education - Ho Chi Minh City, Viet Nam in 2012 and Inje University, South Korea in 2014, respectively. He is currently working toward the $\mathrm{PhD}$. degree in nano device engineering at the Department of Nanosience and Engineering, Inje University, South Korea. His research focuses on power management IC, DC-DC and AC-DC LED driver ICs.

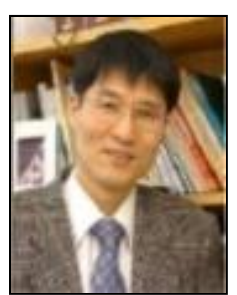

Hanjung Song received the B.S., M.S. and PhD. degrees in electronics engineering from Hanyang University, South Korea in 1986, 1988 and 2000, respectively. In 1998, he was with LG Electronics, Korea. Since 2004, he has been with Inje University, South Korea, where he is a professor. Currently, he is the chairman of the Department of Nanoscience and Engineering, Inje University. His research interests are analog circuit design, semiconductor device modeling, and neural network hardware implementation. 